\title{
Study on Preparation and Stability of Perovskite Solar Cells with Supramolecular Interaction
}

\author{
Qing Zhu ${ }^{1}$, Chengsheng $\mathrm{Li}^{1}$ \\ ${ }^{1}$ Department of Chemical Engineering of Chengxian College in Southeast University, Nanjing, Jiangsu 210088, China
}

\begin{abstract}
In recent years, perovskite solar cells have been developed rapidly because of its excellent crystalline properties, high optical absorption coefficient, high carrier mobility, long carrier life and direct band gap, and suitable to be used as light absorbing layer material of photovoltaic devices. However, the most prominent problem of perovskite materials is that a lot of organic cations will migrate and lose in the process of illumination or heating, which is considered to be the main reason for the performance degradation of perovskite films and devices. Therefore, the suppression of ion migration in perovskite is helpful to improve the stability of perovskite materials and devices and enhance their photoelectric properties. In order to effectively control the ion migration in perovskite films, this paper uses the cation- $\pi$ supramolecular interaction, to prepare the perovskite films with better photoelectric performance and higher stability. The experimental results show that the photoelectric conversion efficiency of the perovskite solar cellsdevice doped with rubrene is increased from $18.60 \%$ to $20.86 \%$, and the hysteresis of the cell is also significantly suppressed.
\end{abstract}

\section{Introduction}

Perovskite solar cells have the characteristics of low cost and high performance, and their photoelectric conversion efficiency has been greatly improved in a few years. Perovskite materials have attractive photoelectric properties, but they also have many disadvantages. The most prominent problem is that there will be a lot of migration and loss of organic cations in the process of illumination or heating. This phenomenon of ion migration is considered to be the main reason for the performance degradation of perovskite films and devices, and it can not be solved by means of packaging or adjusting components. Therefore, the phenomenon of ion migration in perovskite is a problem of perovskite material itself, which seriously affects the stability of perovskite films and devices.

In perovskite materials, there are two kinds of ions that are easy to migrate, namely organic cations and halogen ions, and the former is more sensitive to the external environment. By applying polarization field to perovskite film, organic cations will migrate obviously under the action of polarization field, which can change the chemical and electrical equilibrium state of perovskite film. Under the condition of light, the interaction energy of hydrogen bond between organic cation and $\mathrm{Pb}-\mathrm{I}$ framework will be weakened, which makes the organic cation more likely to migrate; the large-scale migration of organic cations will result in a large number of ion dislocation and defect states, which will accelerate the ion migration, cause the loss of cations in perovskite materials, and cause the rapid attenuation of perovskite films and devices. Therefore, the inhibition of ion migration in perovskite materials is helpful to improve the stability of perovskite materials and devices and enhance their photoelectric properties.

In this paper, in order to effectively control the ion migration in perovskite films, we use the cation- $\pi$ supramolecular interaction, which is widely used in the supramolecular system, in an innovative way by doping the rubrene molecules into the perovskite films. We found that the cation- $\pi$ interaction between rubrene and perovskite can effectively limit the migration of cations in perovskite, inhibit the generation of defects and improve the stability of perovskite films. Through this method, we have prepared more stable perovskite films, and the corresponding battery devices also show excellent photoelectric performance and stability.

\section{Formation and Mechanism of Supramolecular Interaction}

Generally speaking, the cation - $\pi$ interaction is a noncovalent interaction, which is produced between $\pi$ body of electron rich and adjacent cations. Therefore, for the fluorine-doped Perovskite, the interaction exists between aromatic $\pi$ - body molecules and perovskite organic cations. In order to find out the interaction characteristics of the cation $\pi$ - body between rubrene and perovskite, we use density functional theory (DFT) to simulate and calculate the interaction energy between $\mathrm{Ma}^{+}$ cation and rubrene. Considering that $\mathrm{Ma}^{+}$cation can interact with different $\pi$ - body of rubrene, through the optimization of the maximum interaction energy, we get 
the optimal relative position between $\mathrm{Ma}^{+}$cation and rubrene.

As shown in Figure 1(a), there is a chelation like interaction between $\mathrm{MA}^{+}$cations and rubrene: the two N$\mathrm{H}$ bonds of $\mathrm{MA}^{+}$cations are oriented towards the rubrene molecule, and through $\sigma_{N-H} *-\pi_{\text {rubrene }}$, they interact with the p-tetraphenyl center and the phenyl group of the rubrene respectively, so their $\mathrm{N}-\mathrm{H}$ bonds are longer than other N-H bonds.

In order to further study this interaction, we have drawn the molecular electronic potential distribution, as shown in Figure 1(b). It can be seen that the surface of $\mathrm{MA}^{+}$cations is covered by positrons, which makes the polarized $\pi$ electrons close to the $\mathrm{MA}^{+}$cation and rubrene attract each other. It is worth noting that the interaction energy of $\mathrm{MA}^{+}$cation and rubrene is $-35.4 \mathrm{kcal} / \mathrm{mol}$, which is almost twice that of common cation $\pi$. This strong interaction can firmly fix the easily migrated $\mathrm{MA}^{+}$ cations, thereby inhibiting the migration of organic cations in the perovskite, as shown in Figure 1(c).

In order to further prove the interaction between rubrene and perovskite, we used Fourier Transform Infrared Spectrometer (FTIR) to test the perovskite film with and without rubrene and the sample of rubrene film, and compared the FTIR spectra of the above samples. As shown in Figure 1(d), the FTIR spectra of the perovskite films without rubrene show obvious absorption peak at $908 \mathrm{~cm}^{-1}, 1350 \mathrm{~cm}^{-1}, 1465 \mathrm{~cm}^{-1}, 1714 \mathrm{~cm}^{-1}$ and $3132 \mathrm{~cm}^{-1}$. These peaks reveal the vibration of $\mathrm{C}-\mathrm{H}$ and $\mathrm{N}-\mathrm{H}$ bonds in organic groups in perovskite films. For the FTIR spectra of the film, we can see that there are obvious peaks of C$\mathrm{C}$ bond and $\mathrm{C}=\mathrm{C}$ bond of the film. However, for the perovskite films doped with rubrene, all the characteristic peaks of perovskite and rubrene, such as $\mathrm{C}-\mathrm{H}, \mathrm{N}-\mathrm{H}, \mathrm{C}-\mathrm{C}$ and $\mathrm{C}=\mathrm{C}$ bonds, almost disappeared. It shows that the introduction of rubrene can form cation $\pi$ interaction, which can significantly inhibit the movement of organic groups in perovskite, consistent with DFT calculation results. (a)
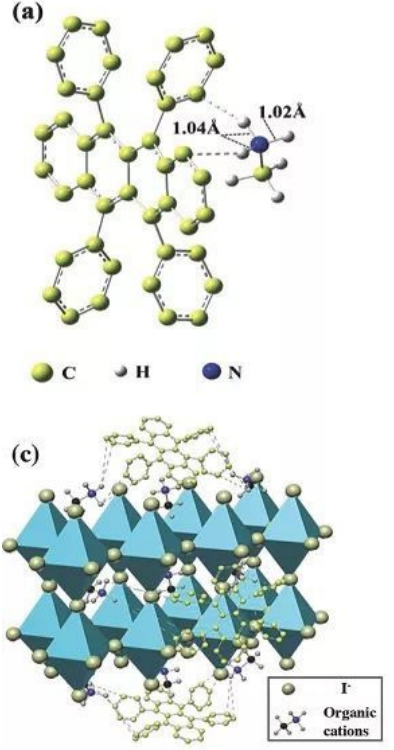

(b)

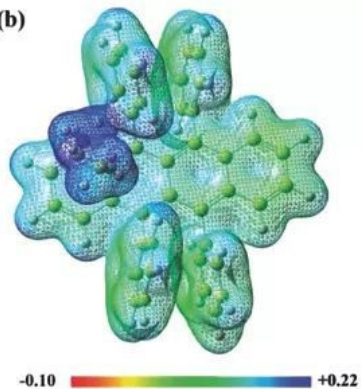

(d)

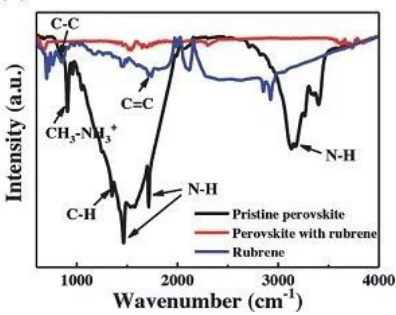

Figure 1. Supermolecular interaction mechanism of perovskite and rubrene

(a) DFT calculation diagram of the interaction between $\mathrm{MA}^{+}$ cation and rubrene

(b) Potential distribution of interaction between $\mathrm{MA}^{+}$cation and rubrene

(c) Schematic diagram of cation $\pi$ interaction between rubrene and perovskite

(d) FTIR spectra of perovskite films with and without rubrene and pure rubrene

\section{Preparation of Perovskite Solar Cells with Supramolecular Interaction}

\subsection{Experimental Materials}

The experimental reagents and materials used in this paper is shown in Table 1.

Table 1. Experimental reagents and materials

\begin{tabular}{|c|c|c|c|}
\hline Reagent name & Molecular formula & Purity & Manufacturer \\
\hline Lead iodide & $\mathrm{PbI}_{2}$ & $99.999 \%$ & Sigma-Aldrich \\
\hline Methylammonium iodide & $\mathrm{CH}_{6} \mathrm{IN}$ & $99.5 \%$ & $\begin{array}{c}\text { Xi'an Polymer Light } \\
\text { Technology Corp. }\end{array}$ \\
\hline Titanium diisopropoxide & $\mathrm{C}_{16} \mathrm{H}_{28} \mathrm{O}_{6} \mathrm{Ti}$ & Superior pure & Aladdin \\
\hline $\begin{array}{c}\text { Titanium dioxide mesoporous layer } \\
\text { slurry }\end{array}$ & $\mathrm{TiO}_{2}$ & $/$ & Deysol \\
\hline Titanium tetrachloride & $\mathrm{TiCl}_{4}$ & Superior pure & Aladdin \\
\hline Tin oxide colloid & $\mathrm{SnO}_{2}$ & Superior pure & Alfa-Aesar \\
\hline$\beta$-Cyclodextrin & $\mathrm{C}_{42} \mathrm{H}_{70} \mathrm{O}_{35}$ & $99 \%$ & Alfa-Aesar \\
\hline Formamidine iodide & $\mathrm{CH}_{5} \mathrm{IN}_{2}$ & $99.5 \%$ & $\begin{array}{c}\text { Xi'an Polymer Light } \\
\text { Technology Corp. }\end{array}$ \\
\hline Rubrene & $\mathrm{C}_{42} \mathrm{H}_{28}$ & $98 \%$ & Sigma-Aldrich \\
\hline Titanium dioxide nanoparticles & $\mathrm{TiO}_{2}$ & $99.8 \%$ & Aladdin \\
\hline Dimethyl sulfoxide & $\mathrm{C}_{2} \mathrm{H}_{6} \mathrm{OS}$ & Superior pure & Sigma-Aldrich \\
\hline
\end{tabular}




\begin{tabular}{|c|c|c|c|}
\hline Spiro-OMeTAD & $\mathrm{C}_{81} \mathrm{H}_{68} \mathrm{~N}_{4} \mathrm{O}_{8}$ & Superior pure & $\begin{array}{c}\text { Xi'an Polymer Light } \\
\text { Technology Corp. }\end{array}$ \\
\hline 4-tert-butylpyridine & $\mathrm{C}_{9} \mathrm{H}_{13} \mathrm{~N}$ & Superior pure & Sigma-Aldrich \\
\hline $\mathrm{N}, \mathrm{N}$ - dimethylformamide & $\mathrm{C}_{3} \mathrm{H}_{7} \mathrm{NO}$ & Superior pure & $\begin{array}{c}\text { Xi'an Polymer Light } \\
\text { Technology Corp. }\end{array}$ \\
\hline Chlorobenzene & $\mathrm{C}_{6} \mathrm{H}_{5} \mathrm{Cl}$ & Superior pure & Sigma-Aldrich \\
\hline Acetonitrile & $\mathrm{C}_{2} \mathrm{H}_{3} \mathrm{~N}$ & Superior pure & Sigma-Aldrich \\
\hline Isopropanol & $\mathrm{C}_{3} \mathrm{H}_{9} \mathrm{O}$ & Superior pure & Sigma-Aldrich \\
\hline
\end{tabular}

\subsection{Preparation of Spin Coating Solution}

First, prepare the electron transmission spin coating solution. Take $1 \mathrm{ml} \mathrm{SnQ} Q_{2}$ hydrogel precursor solution, add $6 \mathrm{ml}$ high purity water according to the volume ratio of $1: 6$. Then, filter the mixture by $0.45 \mu \mathrm{m}$ water filter and pour it into a small bottle after stirring for 30 minutes, then the spin coating solution of $\mathrm{SnO}_{2}$ electron transmission spin coating solution is obtained.

Second, prepare the perovskite spin coating solution. Weigh $0.15 \mathrm{~mol}$ Formamidine iodide, $0.85 \mathrm{~mol}$ Methyliodide amine and $1.025 \mathrm{~mol} \mathrm{PbI}_{2}$, and pour it into the mixture of N, N - Dimethylformamide and Dimethyl sulfoxide, and stir in the glove box for 4 hours to prepare a perovskite spin coating solution.

Third, prepare the rubrene chlorobenzene solution. Take a certain weight of rubrene, and add it to chlorobenzene in proportion to the concentration $(0-20$ $\mathrm{mg} / \mathrm{ml}$ ), and then stir in the glove box for 30 minutes to obtain different concentrations of rubrene chlorobenzene solution.

Finally, prepare the Spiro-OMeTAD hole transport layer spin coating solution. Take 79mg Spiro-OMeTAD and add to $1 \mathrm{ml}$ chlorobenzene. Stir it until the solution becomes transparent, and add $17.5 \mu \mathrm{l}$ prepared lithium salt solution and $28.5 \mu \mathrm{l}$ 4-tert-butylpyridine (TBP), and stir in the glove box for 4 hours to obtain Spiro-OMeTAD hole transport layer spin coating solution.

\subsection{Preparation of Perovskite Solar Cells}

Firstly, treat the cleaned ITO glass with ozone, and spin the prepared $\mathrm{SnO}_{2}$ spin coating liquid on the ITO basal. Then, transfer the sample to the heating plate, heat it at $150^{\circ} \mathrm{C}$ for $30 \mathrm{~min}$ in the air environment, cool it down with the furnace after heating, and ozone the prepared $\mathrm{SnO}_{2}$ film sample again. Then, spin on perovskite spin coating solution on the substrate and when the spin coater works to the eighth second, rinse the surface of the perovskite film with the prepared rubrene chlorobenzene solution of different concentrations to obtain the perovskite film doped with rubrene. Put the sample on the heating table at $130^{\circ} \mathrm{C}$ and anneal for $10 \mathrm{~min}$. And then, coate the solution on the calcium titanium ore layer to obtain the hole transport layer. Finally, prepare gold electrode by vacuum evaporation. The thickness of $\mathrm{Au}$ electrode is about $100 \mathrm{~nm}$, and then we can get perovskite solar cells with supramolecular interaction.

\section{Performance and Stability of Perovskite Solar Cells with Supramolecular Interaction}

\subsection{Photoelectric Performance}

It is found that the interaction of cation $\pi$ between rubrene and perovskite has a significant effect on improving the photoelectric properties of perovskite films and inhibition of ion migration in thin films. Therefore, the perovskite solar cells doped with rubrene should also have higher efficiency and better stability. In this paper, we study the effect of different concentration $(0-20 \mathrm{mg} / \mathrm{ml})$ of rubrene on the performance of perovskite solar cells.

We select 20 battery devices from each doping concentration, and compare the average value of the photoelectric conversion parameters of these devices at different doping concentrations, as shown in Table 2. It can be seen that the performance of perovskite solar cells at different doping concentrations shows a trend of rising first and then decreasing, which shows that the properties of the perovskite battery can be significantly affected by the addition of rubrene into the perovskite, even if the concentration is very low. By comparing these data, we get the best concentration of rurene is $10 \mathrm{mg} / \mathrm{ml}$.

Table 2. Battery performance with different concentration of

\begin{tabular}{|c|c|c|c|c|}
\hline $\begin{array}{c}\text { Concentrations } \\
\text { of rubrene in } \\
\text { chlorobenzene }\end{array}$ & $\begin{array}{c}\mathrm{J}_{\mathrm{SC}} \\
(\mathrm{mA} \\
\left.\mathrm{cm}^{-2}\right)\end{array}$ & $\begin{array}{c}\mathrm{V}_{\mathrm{OC}} \\
(\mathrm{V})\end{array}$ & $\begin{array}{c}\mathrm{FF} \\
(\%)\end{array}$ & $\begin{array}{c}\text { PCE } \\
(\%)\end{array}$ \\
\hline $\begin{array}{c}\text { Standard }(0 \\
\mathrm{mg} / \mathrm{ml})\end{array}$ & 22.52 & 1.08 & 72.42 & 17.66 \\
\hline $5 \mathrm{mg} / \mathrm{ml}$ & 22.68 & 1.07 & 70.64 & 17.14 \\
\hline $10 \mathrm{mg} / \mathrm{ml}$ & 23.80 & 1.11 & 76.90 & 20.39 \\
\hline $15 \mathrm{mg} / \mathrm{ml}$ & 23.76 & 1.11 & 77.12 & 20.33 \\
\hline $20 \mathrm{mg} / \mathrm{ml}$ & 22.22 & 1.09 & 75.07 & 18.28 \\
\hline
\end{tabular}

As shown in Figure 2(a), when the concentration of rubrene is $10 \mathrm{mg} / \mathrm{ml}$, the highest efficiency of the perovskite solar cells is $20.86 \%, 12 \%$ higher than that of the solar cells without rubrene. In addition, we tested the photocurrent and efficiency of perovskite solar cells at the 
maximum power point, as shown in Figure 2(b). By keeping the bias voltage $(0.95 \mathrm{v})$ during the test, we get a stable efficiency of $20.63 \%$, which is close to the maximum efficiency. It is worth noting that $J_{S C}$ of the two devices changes most obviously when comparing the optimized photoelectric conversion parameters of the
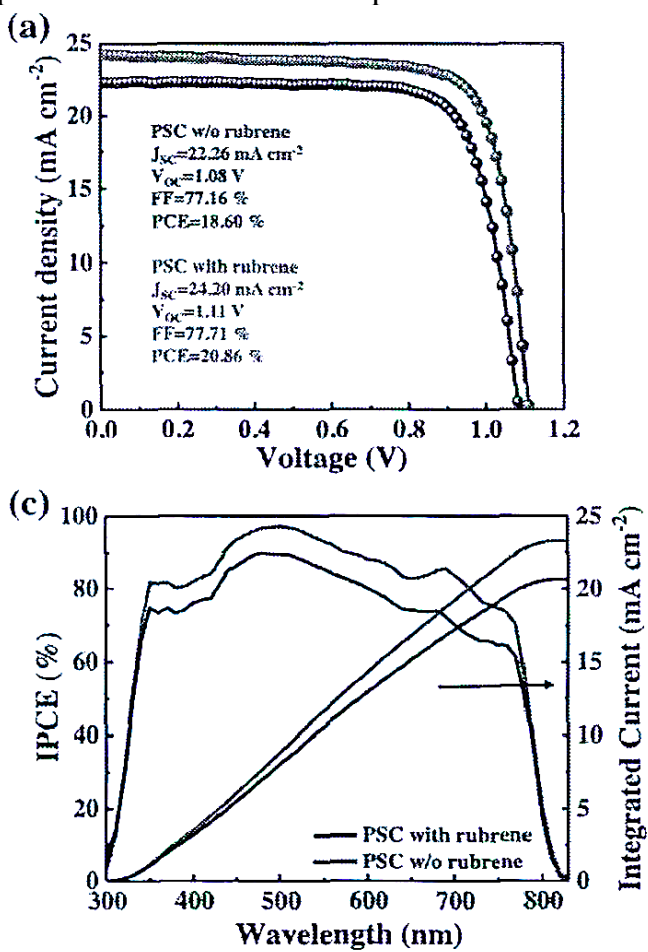

devices with and without the addition of rubrene. In order to find out the reason for the enhancement of perovskite devices $J_{S C}$ after adding rubrene, we measured the external quantum efficiency (EQE) of the two devices respectively, as shown in Figure 2(c).
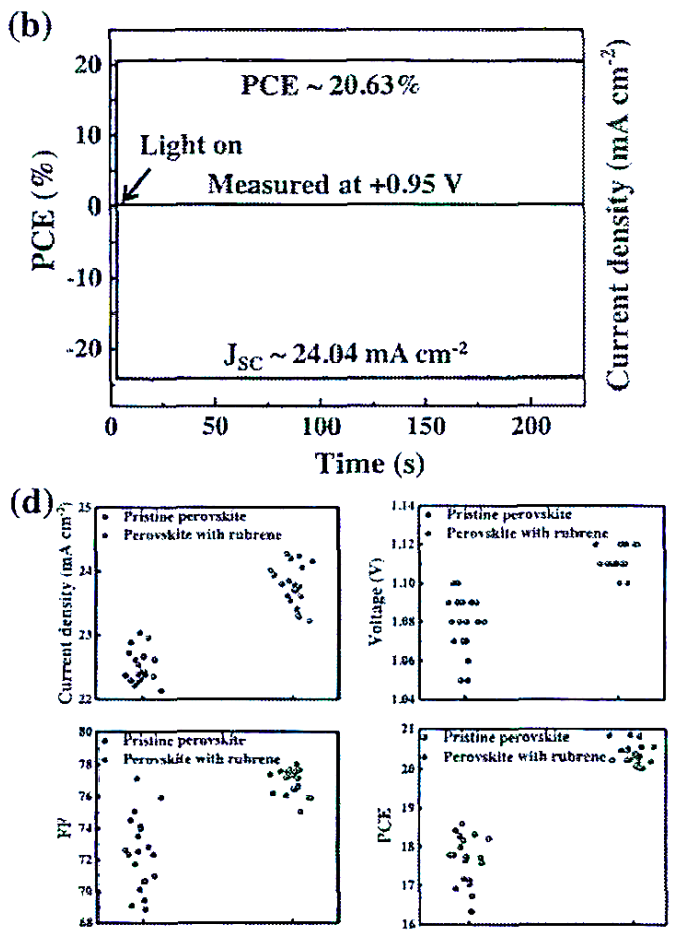

Figure 2. The improvement of the performance of perovskite solar cells by adding rubrene

(a) The highest efficiency curve of perovskite solar cells with and without rubrene;

(b) The steady-state photocurrent and efficiency test curve at the maximum power point of the perovskite solar cells doped with rubrene;

(c) EQE curve and fitting photocurrent of perovskite solar cells with and without rubrene;

(d) Distribution of photoelectric conversion parameters of cells with and without rubrene.

In can be seen from Figure 2 that, the EQE of the perovskite cell doped with rubrene is larger than that of the device without rubrene in the whole visible light range. Moreover, the $\mathrm{J}_{\mathrm{SC}}$ fitted by $\mathrm{EQE}$ curve is in good agreement with the actual $\mathrm{J}_{\mathrm{SC}}$ measured. The results indicate that the carrier transport efficiency of perovskite solar cells is improved by adding rubrene, and the corresponding external quantum efficiency and the device short-circuit current and other photoelectric conversion parameters are significantly improved.

At the same time, we also examined the repeatability of the performance of perovskite solar cells with and without rubrene, as shown in Figure 2(d). It can be seen that, compared with the cell device without rubrene, the perovskite solar cells with rubrene has better repeatability. The photoelectric conversion efficiency of the cell without adding rubrene shows a large distribution, from $16.33 \%$ to $18.6 \%$; while the distribution of photoelectric conversion parameters of devices doped with rubrene is more concentrated, and the cell efficiency is distributed between $20.02 \%$ and $20.86 \%$.

\subsection{Stability}

For perovskite solar cells, in addition to the high photoelectric conversion efficiency, the stability of the battery device in the working environment is also an important factor to measure the performance of the device. In order to study the stability of perovskite solar cells with and without rubrene, we tested the above devices every 3 days under AM1.5G light, continuous testing for one month. During the test interval, we placed the tested device in a glass dish with a humidity of $75 \%$ and a temperature of $25^{\circ} \mathrm{C}$, protected from light. As shown in Figure 3(a), the perovskite solar cells doped with rubrene show excellent long-term stability. After long-term testing, the efficiency of the battery device doped with rubrene changed from $20.57 \%$ to $20.35 \%$, still retaining $98 \%$ of the original efficiency. For devices without rubrene, the battery efficiency dropped from $17.41 \%$ to $4.45 \%$, which indicates that the stability of the device without doped rubrene is poor, and severe attenuation occurs during the long-term light test. As shown in Figure 3(b) and Figure 3 (c), we have drawn the $J-V$ curve of the above two batteries after 30 days of long-term test and untested. The illustrations are digital photos of these two batteries after 30 days of testing, which were taken from the glass 
surface of the device. It can be clearly seen from the figure that after 30 days of light test, the perovskite battery device not doped with rubrene shows an off-white color in the photoelectrically active area, and through the entire device, we can clearly see the gold electrode. The perovskite solar cells doped with rubrene still maintains a red-brown color, indicating that the photoelectric active layer of this type of cell still retains its original optical properties and has not changed. These results indicate that the perovskite battery doped with rubrene has better long- term stability, and this excellent characteristic is mainly due to the cation- $\pi$ interaction between rubrene and perovskite. This interaction inhibits the ion migration caused by the perovskite device under long-term light conditions, thereby reducing the concentration of defect states generated on the surface and grain boundaries of the perovskite due to ion migration, and as a result, the attenuation of perovskite films and battery devices is greatly reduced.

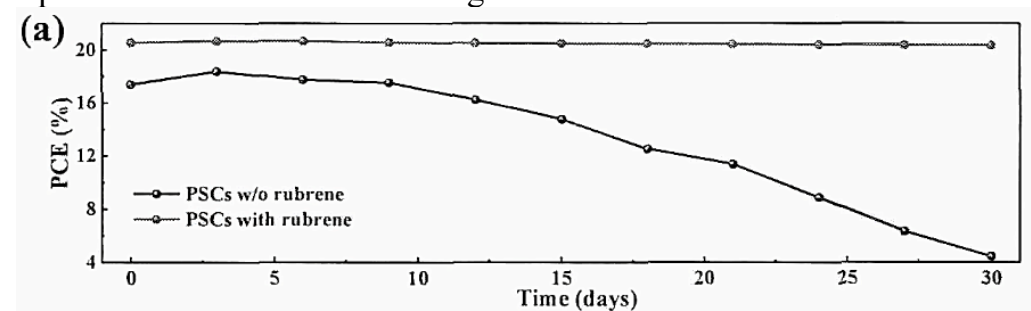

(b)

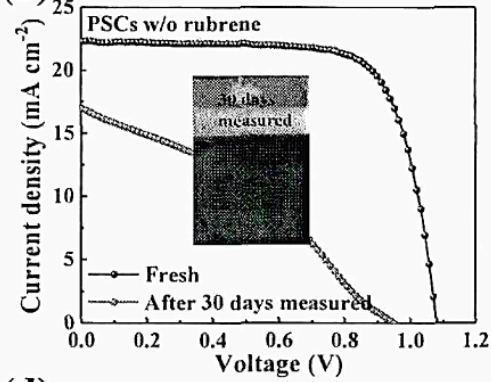

(d)

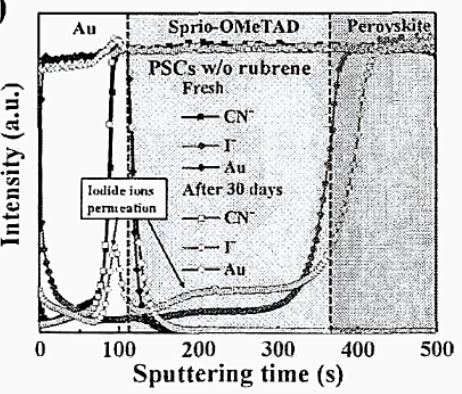

(c)

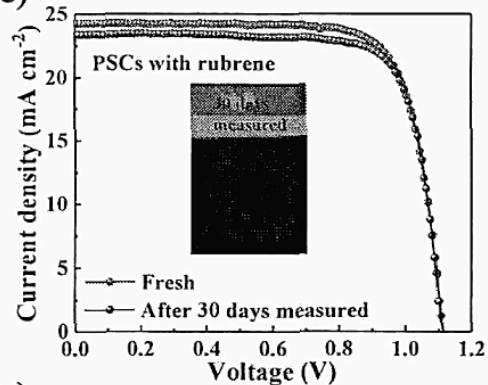

(e)

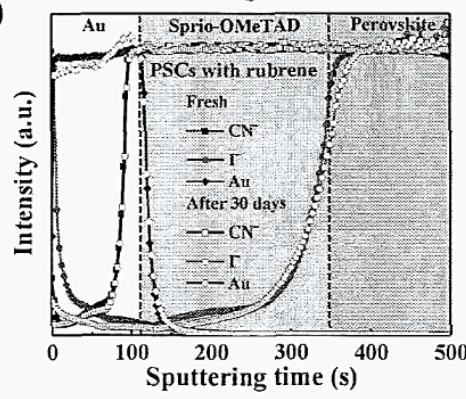

Figure 3. Stability test of perovskite with and without rubrene

(a) Long-term stability test curve of perovskite solar cells with and without rubrene;

(b) $J-V$ curve before and after the long-term stability test of the perovskite solar cells not doped with rubrene;

(c) $J-V$ curve before and after the long-term stability test of the perovskite solar cells doped with rubrene;

(d) Comparison of TOF-SIMS before and after long-term stability test of perovskite solar cells without rubrene;

(e) Comparison of TOF-SIMS before and after long-term stability test of perovskite solar cells with rubrene.

In order to find out the cause of the attenuation of the perovskite battery device during the long-term stability test, we used TOF-SIMS to characterize the changes of the elements and groups of the above devices before and after the 30-day long-term stability test. Here we examine the changes of $\mathrm{CN}^{-}, \mathrm{I}^{-}$and $\mathrm{Au}$ elements in the above two perovskite devices with ion beam sputtering time (from 0 to 500 seconds), as shown in Figure 3(d) and Figure 3(e). It can be seen that, for perovskite solar cells not doped with rubrene, after a long-term stability test, the I-ions in the perovskite thin film have diffused into the SpiroOMED layer and the Au electrode layer in large amounts, while this is not the case for perovskite devices doped with rubrene. This is because during the preparation and test of the perovskite films without rubrene, a large number of defects and vacancies are caused on the surface and grain boundaries of the films due to the ion migration and the loss of organic groups, and these defects and vacancies become the channels of ion migration, which leads to more serious phenomenon of ion migration in perovskite films. Under the influence of cation - $\pi$ interaction, the organic groups in the films are firmly fixed, and the defects at the grain boundary are effectively passivated, so there are fewer defects and vacancies at the surface and grain boundaries, which reduces the ion migration channel and improves the stability of the perovskite film. The application of this perovskite film with ion migration resistance to battery devices can greatly improve battery performance and significantly enhance the long-term stability of perovskite solar cells. 


\section{Conclusion}

In this paper, we apply the cation - $\pi$ interaction to the perovskite material to inhibit the migration of organic groups in the material, so as to obtain the perovskite film with less defect density of state and more stable lattice structure. By applying the film to solar cellsdevices, we have obtained perovskite solar cellsdevices with the highest efficiency of $20.86 \%$ and long-term stability. In addition, the perovskite film with suppressed ion migration has fewer migration channels, which can effectively reduce the migration of iodine ions in perovskite, so as to protect the interface between perovskite and the transport layer from damage caused by ion migration and improve the stability of the device. Therefore, the perovskite solar cells doped with rubrene show excellent stability, after 720 hours of long-term stability test, they still maintain $98 \%$ of the original efficiency.

\section{References}

1. Abate A, Saliba M, Hollman D J, et al. Supramolecular Halogen Bond Passivation of Organic-Inorganic Halide Perovskite Solar Cells[J]. Nano Letters, 2014, 14(6):3247-3254.

2. Dong, Wei, Hao, et al. Moisture-tolerant supermolecule for the stability enhancement of organic-inorganic perovskite solar cells in ambient air.[J]. Nanoscale, 2019, 23(002):105-146.

3. Milic J V , Im J H , Kubicki D J , et al. Supramolecular Engineering for Formamidinium-Based Layered 2D Perovskite Solar Cells: Structural Complexity and Dynamics Revealed by Solid-State NMR Spectroscopy[J]. Advanced energy materials, 2019, 9(20):1900284.1-1900284.12.

4. Song J , Bian J , Zheng E, et al. Efficient and Environmentally Stable Perovskite Solar Cells Based on $\mathrm{ZnO}$ Electron Collection Layer[J]. Chemistry Letters, 2015, 44(5):610-612.

5. Saygili Y, Turren-Cruz S H, Olthof S, et al. Planar Perovskite Solar Cells with High Open-Circuit Voltage Containing a Supramolecular Iron Complex as Hole Transport Material Dopant[J]. Chemphyschem, 2018,56(13):87-109.
6. Li M, Li Y, Sasaki S I, et al. Dopant-Free Zinc Chlorophyll Aggregates as an Efficient Biocompatible Hole Transporter for Perovskite Solar Cells[J]. Chemsuschem, 2016, 27(018):32-54.

7. Ion-Migration Inhibition by the Cation- $\pi$ Interaction in Perovskite Materials for Efficient and Stable Perovskite Solar Cells[J]. Advanced Materials, 2018, 30(31):1707583.1-1707583.10.

8. Ladder-like conjugated polymers used as holetransporting materials for high-efficiency perovskite solar cells[J]. Journal of Materials Chemistry A, 2019, 7(005):44-57.

9. Emilio, Jose, Palomares, 等 . Supramolecular Coordination of $\mathrm{Pb} 2+$ Defects in Hybrid Lead Halide Perovskite Films Using Truxene Derivatives as Lewis Base Interlayers.[J]. Chemphyschem A European Journal of Chemical Physics \& Physical Chemistry, 2019,5(002):143-186.

10. Liu D, Kelly T L . Perovskite solar cells with a planar heterojunction structure prepared using roomtemperature solution processing techniques[J]. Nature Photonics, 2013, 8(2):133-138.

11. You J, Hong Z, Yang Y, et al. Low-Temperature Solution-Processed Perovskite Solar Cells with High Efficiency and Flexibility[J]. Acs Nano, 2014, 8(2):1674-1680.

12. Hao F, Stoumpos C C , Cao D H , et al. Lead-free solid-state organic-inorganic halide perovskite solar cells[J]. Nature Photonics, 2014, 8(6):489-494.

13. Wanyi, Nie, Hsinhan,等. Solar cells. High-efficiency solution-processed perovskite solar cells with millimeter-scale grains.[J]. Science, 2015, 7(3):274288.

14. Wojciechowski K, Saliba M, Leijtens T, et al. Sub$150{ }^{\circ} \mathrm{C}$ processed meso-superstructured perovskite solar cells with enhanced efficiency[J]. Energy \& environmental science, 2014, 7(3):1142-1147.

15. Kim H S , Park N G . Correction to "Parameters Affecting I - V Hysteresis of $\mathrm{CH} 3 \mathrm{NH} 3 \mathrm{PbI} 3$ Perovskite Solar Cells: Effects of Perovskite Crystal Size and Mesoporous TiO 2 Layer"[J]. Journal of Physical Chemistry Letters, 2014, 5(17):2927-2934. 